\title{
Sistema de control para procesos de producción
}

\author{
Javier Jiménez-Cabas, Jorge Cárdenas-Cabrera, Andrés Polo-Panza \\ Departamento de Ciencias de la Computación y Electrónica, Universidad de la Costa, Barranquilla, Colombia. \\ jjimenez41@cuc.edu.co,jcardenas@cuc.edu.co,apolo36@cuc.edu.co
}

Recibido: Febrero 20, 2020.

Recibido en su versión corregida: Mayo 12, 2020.

Aceptación: Junio 02, 2020.

Cómo citar: Jiménez-Cabas, J., Cárdenas-Cabrera, J. \& Polo-Panza, A. (2020). Sistema de control para procesos de producción. Revista Sextante, 23, pp. 1 - 11, 2020.

\section{Resumen}

En este artículo se desarrolla una estrategia de control para un proceso de producción, conformado por un reactor con calentamiento, adquirido a través de un serpentín con vapor. Además de la variable controlada que es la concentración del producto, $C_{B}$, se controlan adicionalmente el nivel y la temperatura. Se sintonizaron los controladores PID, empleando el procedimiento del modelo de primer orden más tiempo muerto (FOPDT). Finalmente, se realizaron varias simulaciones bajo condiciones de operaciones diferentes. La primera prueba realizada consistió en operar el sistema en condiciones nominales, haciendo $C_{B}^{\text {set }}=0.4$. Se observa un sobre-pico de 0.55\% para la concentración de B, que se considera bastante bajo, en cuanto al tiempo de establecimiento se observó una reducción considerable, se pasó de 30 min en un lazo abierto a 15 min en un lazo cerrado; adicionalmente, se logra un error en estado estacionario nulo con lo que se verifica la efectividad del lazo de control.

Palabras clave: Balance de energía; Flujo; Nivel; Reactor; Temperatura; Vapor.

\section{Control System for Production Processes}

\begin{abstract}
In this article, a control strategy for a production process will be developed, consisting of a heated reactor acquired through a steam coil. Besides the controlled variable that is the concentration of the product, $C_{B}$, the level and the temperature are additionally controlled. The PID controllers were tuned using the procedure of the first order model plus dead time (FOPDT). Finally, several simulations were carried out under different operating conditions. The first test carried out consisted of operating the system under nominal conditions, making $C_{B}^{\text {set }}=0.4$. An overshoot of $0.55 \%$ for the concentration of $\mathrm{B}$, which is considered quite low, in terms of the establishment time a considerable reduction was observed, it went from $30 \mathrm{~min}$ in an open loop to $15 \mathrm{~min}$ in a closed loop; additionally, a null steady state flaw is achieved with which the effectiveness of the control loop is verified.
\end{abstract}

Keywords: Energy balance; Flow; Level; Reactor; Steam; Temperature. 


\section{Requisitos del sistema}

\subsection{Características de las variables}

A continuación, se presenta el diseño y la implementación de un sistema de control para un proceso de producción (ver Figura 1), conformado por un reactor con calentamiento adquirido a través de un serpentín con vapor, en el que tienen lugar dos reacciones. La estrategia de control propuesta debe cumplir las siguientes especificaciones:

- La variable controlada es la concentración del producto, $\mathrm{C}_{\mathrm{B}}$.

- La variable manipulada es el flujo de agua.

- Se tienen reportes de variaciones súbitas en la presión de suministro de agua al reactor.

- $\quad$ Si el nivel del líquido aumenta por encima de 8 pies, el flujo másico total debe ser reducido.

- Si la temperatura del reactor aumenta por encima de $190^{\circ} \mathrm{F}$, la relación agua/carga debe incrementarse. Si dicha relación alcanza 5:1 y aun no se reduce la temperatura por debajo de $190^{\circ} \mathrm{F}$, debe reducirse al suministro de vapor. (La relación carga/agua máxima es 3:1).

\section{Descripción del proceso}

El sistema mostrado en la Figura 1 corresponde a un reactor en el que tienen lugar simultáneamente las siguientes dos reacciones:

$$
\begin{aligned}
& \text { (1): } 2 A \rightarrow B+C \\
& \text { (2): } 2 C \rightarrow B+E
\end{aligned}
$$

En la Ecuación 1, la reacción (1) es endotérmica, con un calor de reacción obtenido a través del serpentín, mientras que el calor para la reacción (2) es despreciable. Las velocidades de reacción están dadas por la Ecuación 2:

$$
\begin{gathered}
r_{A}(t)=k_{0} c_{A}(t) c_{B}(t) e^{\frac{-E}{R T(t)}} \\
r_{C}(t)=k_{0}^{\prime} c_{A}(t) c_{C}(t)
\end{gathered}
$$

Por su parte, el flujo de la válvula de salida está dado por la Ecuación 3:

$$
f(t)=C_{V} \sqrt{h(t)}
$$

Las densidades en la corriente de alimentación, el reactor y en la corriente de salida son función de la concentración de B (Ecuación 4):

$$
\rho(t)=\rho_{0}+d c_{B}(t)
$$

El encargado de la planta ha indicado que una de las principales fuentes de perturbación es la concentración en la corriente de alimentación, $\operatorname{cAi}(t)$.

Los parámetros y condiciones iniciales del proceso se muestran en la Tabla 1.

\section{Modelo dinámico}

A continuación, se presentan los distintos balances que se establecen para el sistema:

\subsection{Balance global de masa:}

$$
A\left(\frac{d}{d t}\right)(h(t) \rho(t))=\rho_{1} F_{1}(t)+\rho_{2} F_{2}(t)-\rho(t) F(t)
$$

\subsection{Balances de energía:}

\section{Reactor:}

$$
\begin{aligned}
A c_{v} \frac{d}{d t}(h(t) \rho(t) T(t))= & \\
& c_{p p} \rho_{1} T_{1} F_{1}(t)+c_{m w} \rho_{2} T_{2} F_{2}(t)-c_{p} F(t) \rho(t) T(t) \\
& +U_{o} A_{c}\left(T_{c}(t)-T(t)\right)-\Delta H_{m A} A r_{A}(t) h(t)
\end{aligned}
$$

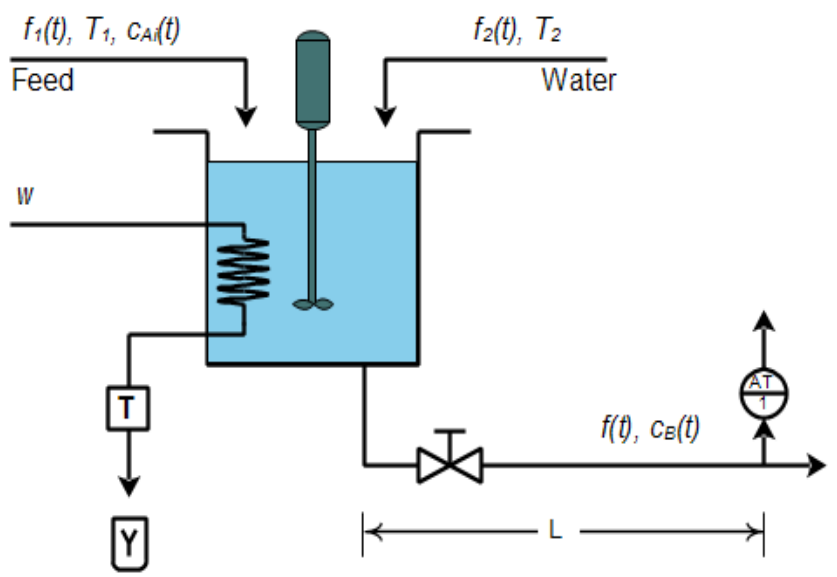

Figura 1. Tanque reactor continuamente mezclado (CSTR).

Fuente: Los autores. 
Serpentín:

$$
c_{p c} w_{c} L_{c} \frac{d}{d t}\left(T_{c}(t)\right)=\lambda W(t)-U_{O} A_{c}\left(T_{c}(t)-T(t)\right)
$$

Para el caso de las tablas, deben ser ajustadas al formato ejemplificado como la Tabla 1, y rotuladas en la parte superior con su respectiva enumeración $\mathrm{y}$ fuente en la parte inferior.

\subsection{Balance de masa por componente:}

Componente A:

$$
\begin{aligned}
A \frac{d}{d t}\left(h(t) c_{A}(t)\right) & \\
& =c_{A i}(t) F_{1}(t)-c_{A}(t) F(t) \\
& -A r_{A}(t) h(t)
\end{aligned}
$$

Componente B:

$$
\begin{aligned}
A \frac{d}{d t}\left(h(t) c_{B}(t)\right) & \\
& =-c_{B}(t) F(t)+0.5 A r_{A}(t) h(t) \\
& +0.5 A r_{c}(t) h(t)
\end{aligned}
$$

Componente $\mathrm{C}$ :

$$
\begin{aligned}
A \frac{d}{d t}\left(h(t) c_{C}(t)\right) & \\
& =-c_{C}(t) F(t) \\
& +0.5 A r_{A}(t) h(t)-A r_{c}(t) h(t)
\end{aligned}
$$

Desde la Ecuación 2 hasta la Ecuación 10 se encuentra descrito de forma matemática el modelo dinámico para el reactor estudiado (ver Figura 1).

\section{Instrumentación}

Las características de los diferentes transmisores necesarios para realizar el control del proceso se determinaron a partir de las hojas de especificaciones de instrumentos disponibles en el mercado, por ejemplo:

Para la medición del nivel y el flujo, se encontró que los transmisores tienen tiempos de respuesta bastantes bajos ( $25 \mathrm{~ms}$ en el caso de nivel), en comparación con los tiempos que se manejan en este proceso, por lo tanto, solo se modelaron como ganancias puras.

Para el sensor de temperatura se estableció un rango de $200{ }^{\circ} \mathrm{F}$, el cual cubre las temperaturas que se van a trabajar en el proceso, con una constante de tiempo de $0.5 \mathrm{~s}$ (característico de catálogos con amplitud de rangos similares).
Para analizar la concentración se tienen en cuenta varios criterios en el momento de elección de un analizador: primero se determina el rango de concentración en el que se va a trabajar, con el fin de definir el tipo de técnica a utilizar para el análisis, para este proceso a condiciones de operación especiales (doble del flujo en estado estable de A y doble de $\mathrm{C}_{\mathrm{Ai}}$, sin flujo de entrada de agua), se determinó un máximo de concentración para $\mathrm{B}$ de 2 lbmol/ft3, tomándose como span entonces 2 $\mathrm{lbmol} / \mathrm{ft} 3$. Adicionalmente es necesario conocer las características de las muestras, debido a que se escogerá qué tipo de propiedad es más adecuada para realizar la detección; para soluciones se utilizan diferentes tipos de análisis entre estos se encuentran: medición de índices de refracción, conductividad, absorción y dependiendo del tipo de solución y la cantidad de componentes de esta se elige el más acertado. Para el proceso en estudio, como no se conoce qué tipos de sustancias son, se escogió análisis por cromatografía liquida, con lo cual se garantiza determinar la concentración de $\mathrm{B}$ y el resto de las especies que se encuentran después de las reacciones, para este tipo de muestras los tiempos de retención en las columnas son aproximadamente 4 min, con lo cual manejamos una constante de tiempo de 0,8 min. Para el vapor se utiliza una válvula de control que permita trabajar en la condición automática mientras no se presente la condición de seguridad, que obliga a cambiar el porcentaje de apertura de esta.

Para el dimensionamiento de la válvula el vapor se trabaja como fluido compresible normalmente, aunque para las condiciones establecidas en el problema, donde la presión y la temperatura de saturación son cercanas a las atmosféricas (17.02 psi y $219.45 \mathrm{~F}$ ), se considera una caída de presión pequeña a través de la válvula, y para estas condiciones el dimensionamiento se puede aproximar a una válvula de fluido de servicio líquido.

Se tomó como flujo máximo de vapor el doble de dicho flujo en estado estacionario, una caída de presión máxima de 4 psi y una mínima de 2 psi (con lo cual se puede aproximar el comportamiento al de una válvula para fluidos de servicio), se calculó CVmax, se obtuvo un $\alpha$ para determinar qué tipo de válvula se iba a elegir, (el valor es bastante bajo 
Tabla 1. Parámetros y condiciones iniciales.

\begin{tabular}{|c|c|c|}
\hline Variable & Símbolo & Valor \\
\hline \multicolumn{3}{|l|}{ Serpentín } \\
\hline Longitud (ft) & Lc & 240 \\
\hline Diámetro externo (in) & $\phi c$ & 4.5 \\
\hline $\begin{array}{l}\text { Coeficiente de transferencia de calor } \\
\left(\text { BTU } /\left(\min \mathrm{ft}^{2}{ }^{\circ} \mathrm{F}\right)\right)\end{array}$ & $\mathrm{U}_{\mathrm{O}}$ & 5.25 \\
\hline Capacidad calorífica $\left(\mathrm{BTU} /\left(\mathrm{lb}^{\circ} \mathrm{F}\right)\right)$ & $\mathrm{c}_{\mathrm{pc}}$ & 0.14 \\
\hline Peso $(\mathrm{lb} / \mathrm{ft})$ & $\mathrm{W}_{\mathrm{c}}$ & 11.2 \\
\hline \multicolumn{3}{|l|}{ Reacciones } \\
\hline Energía de activación (BTU/lbmol) & $\mathrm{E}$ & 27820 \\
\hline $\begin{array}{l}\text { Constante universal de los gases } \\
\left.\left(\text { BTU/(lbmol }{ }^{\circ} \mathrm{R}\right)\right)\end{array}$ & $\mathrm{R}$ & 1.987 \\
\hline $\begin{array}{l}\text { Constante de equilibrio reacción } \\
\left(\mathrm{ft}^{3} /(\text { lbmolB } \min )\right)\end{array}$ & $\mathrm{k}_{0}$ & $8.5 \times 10^{10}$ \\
\hline $\begin{array}{l}\text { Constante de equilibrio reacción } \\
\left(\mathrm{ft}^{3} /(\text { lbmolA } \min )\right)\end{array}$ & $\mathrm{k}_{0}^{\prime}$ & 41 \\
\hline Delta de Entalpia reacción 1 (BTU/lmolA) & $\Delta \mathrm{H}_{\mathrm{rA}}$ & 1000 \\
\hline \multicolumn{3}{|l|}{$\begin{array}{ll}\text { Válvula de salida } \\
\end{array}$} \\
\hline Coeficiente de flujo $\left(\mathrm{ft}^{3} /\left(\min \mathrm{ft}^{0.5}\right)\right)$ & $\mathrm{C}_{\mathrm{V}}$ & 12.4 \\
\hline \multicolumn{3}{|l|}{ Corriente de alimentación } \\
\hline Densidad $\left(\mathrm{lb} / \mathrm{ft}^{3}\right)$ & & 68 \\
\hline $\begin{array}{l}\text { Parámetro de proporcionalidad } \quad\left(\mathrm{lb} /\left(\mathrm{ft}^{3}\right.\right. \\
\left.\left.\mathrm{lbmolB} / \mathrm{ft}^{3}\right)\right)\end{array}$ & $\mathrm{d}$ & 2.4 \\
\hline Capacidad calorífica $\left(\mathrm{BTU} /\left(\mathrm{lbmol}^{\circ} \mathrm{F}\right)\right)$ & $\mathrm{c}_{\mathrm{p} 1}$ & 0.85 \\
\hline \multicolumn{3}{|l|}{ Capacidad calorífica $\left(\mathrm{BTU} /\left(\mathrm{lbmol}{ }^{\circ} \mathrm{F}\right)\right)$} \\
\hline \multicolumn{3}{|l|}{ Corriente de vapor } \\
\hline Calor latente de condensación (BTU/lbm) & $\lambda$ & 966 \\
\hline \multicolumn{3}{|l|}{ Corriente de agua } \\
\hline Capacidad calorífica $\left(\mathrm{BTU} /\left(\mathrm{lbmol}^{\circ} \mathrm{F}\right)\right)$ & $c_{p w}=c_{v w}$ & 1 \\
\hline Densidad $\left(\mathrm{lb} / \mathrm{ft}^{3}\right)$ & $\rho_{\mathrm{w}}$ & 62.4 \\
\hline \multicolumn{3}{|l|}{ Corriente de producto } \\
\hline Capacidad calorífica $\left(\mathrm{BTU} /\left(\mathrm{lbmol}^{\circ} \mathrm{F}\right)\right)$ & $\mathrm{c}_{\mathrm{p}}=\mathrm{c}_{\mathrm{v}}$ & 0.9 \\
\hline \multicolumn{3}{|l|}{ Tubería } \\
\hline Longitud (ft) & $\mathrm{L}$ & 180 \\
\hline Diámetro interno (in) & $\phi$ & 5.8 \\
\hline \multicolumn{3}{|l|}{ Tanque del reactor } \\
\hline Área $\left(\mathrm{ft}^{2}\right)$ & $\mathrm{A}$ & 30 \\
\hline Altura (ft) & $\mathrm{h}_{\max }$ & 25 \\
\hline \multicolumn{3}{|c|}{ Valores en estado estacionario } \\
\hline Flujo alimentación $\left(\mathrm{ft}^{3} / \mathrm{min}\right)$ & $\mathrm{f}_{1}$ & 18 \\
\hline Flujo agua $\left(\mathrm{ft}^{3} / \mathrm{min}\right)$ & $\mathrm{f}_{2}$ & 14 \\
\hline Temperatura alimentación $\left({ }^{\circ} \mathrm{F}\right)$ & $\mathrm{T}_{1}$ & 140 \\
\hline Temperatura agua $\left({ }^{\circ} \mathrm{F}\right)$ & $\mathrm{T}_{2}$ & 85 \\
\hline Concentración alimentación $\left(\mathrm{lbmolA} / \mathrm{ft}^{3}\right)$ & $\mathrm{C}_{\mathrm{Ai}}$ & 0.95 \\
\hline Flujo de vapor (Lbmol/min) & $\mathrm{W}$ & 40 \\
\hline
\end{tabular}

Fuente: Los autores.

(un valor de 2.4) por lo tanto se eligió proporcional), se obtuvo una ganancia: 0.589 . De manera similar se dimensionaron las válvulas de las corrientes de alimentación y de agua. En la Tabla 2 se resumen las características de los transmisores y elementos finales de control empleados.

\section{Estrategias de control}

Aunque las especificaciones de diseño establecen que la variable controlada es la concentración del producto, $\mathrm{c}_{\mathrm{B}}$, hay especificaciones de seguridad que implican controlar variables adicionales como nivel y 
temperatura. Con esto en mente se decidió calcular, en estado estacionario, el arreglo de ganancias relativas (RGA por sus siglas en inglés), con el objeto de determinar las variables manipuladas más apropiadas para cada variable controlada (J. Jiménez-Cabas et al., 2019; Vijayan \& Ashok, 2020). La RGA obtenida se observa en la Ecuación 11.

$$
\left[\begin{array}{c}
h \\
T \\
c_{B}
\end{array}\right]=\left[\begin{array}{ccc}
0.5173 & 0.4837 & -0.0011 \\
0.0228 & -0.0647 & 1.0419 \\
0.4598 & 0.5810 & -0.0408
\end{array}\right]\left[\begin{array}{l}
f_{1} \\
f_{2} \\
w
\end{array}\right]
$$

Entonces, con base en la Ecuación 11 se decidió manipular $f_{1}$ para controlar h, y $f_{2}$ así controlar $C_{B}$. Esta última selección corresponde con la exigencia de diseño planteada. Para el caso de la temperatura es claro que la mejor opción sería manipular el flujo de vapor, w, pero dado que si la temperatura es superior a $190{ }^{\circ} \mathrm{F}$ en primera instancia debe aumentarse la relación agua/carga, se decide manipular el flujo de alimentación, $f_{1}$.

No se seleccionó $f_{2}$ debido a que la ganancia correspondiente en la RGA es negativa (-0.0647), cabe recordar que, si se seleccionan parejas de variables relacionadas a través de ganancias negativas en la RGA, la acción del proceso cambia cuando los demás lazos se abren y cierran, o cuando sus variables manipuladas alcanzan sus valores límite; llevando a inestabilidad en el lazo correspondiente.

Con el objeto de sintonizar los distintos controladores PID se obtuvieron, empleado el procedimiento Fit 3 descrito en (J. Jiménez-Cabas et al., 2019; Smith \& Corripio, 2012), modelos de primer orden más tiempo muerto (FOPDT) que permiten relacionar los distintos pares de variables controladas y manipuladas. La Tabla 3 se muestra los parámetros de dichos modelos.

La estrategia de control implementada se muestra en la Figura 2. En general se emplearon controladores PID de la forma descrita en la Ecuación 12.

$$
G_{C}(s)=K_{P}+\frac{K_{I}}{s}+\frac{K_{D} N}{\frac{N}{s}+1}
$$

A continuación, se procede con al análisis de cada uno de los lazos de control.

\subsection{Control de la concentración de B (Lazo 1)}

En este caso se tomó en consideración el hecho que son de esperarse variaciones súbitas en la presión de suministro de agua al reactor, por lo que se decidió emplear una estrategia de control en cascada con un lazo de control del flujo como esclavo. Dicho controlador, FC/1, el cual se puede observar en la Figura 2, se sintonizó con base en los parámetros de la válvula de la corriente de agua. Para la sintonización del controlador maestro, AC/1, se emplearon los parámetros de la función de transferencia $C_{B}(s) / F_{2}(s)$.

\subsection{Compensación de perturbaciones en la concentración de alimentación (Lazo 2)}

Dado que una de las principales fuentes de perturbación es la concentración en la corriente de alimentación, $C_{A i}(t)$, y siendo esta una variable temprana medible, se optó por complementar el lazo 1 con un control feedforward (AC/2) que compense las variaciones en $C_{A i}(t)$. La estructura de este controlador es: (Druzgalski et al., 2020), donde

$$
\begin{aligned}
& G_{F F C}(s)=-\frac{K_{5}}{K_{T c_{A i}} K_{1}}\left(\frac{\tau_{1} s+1}{\tau_{5} s+1}\right) e^{-\left(t_{05}-t_{01}\right) s} \\
& =0.6\left(\frac{5.6941 s+1}{5.939 s+1}\right) e^{-0.3826 s}
\end{aligned}
$$

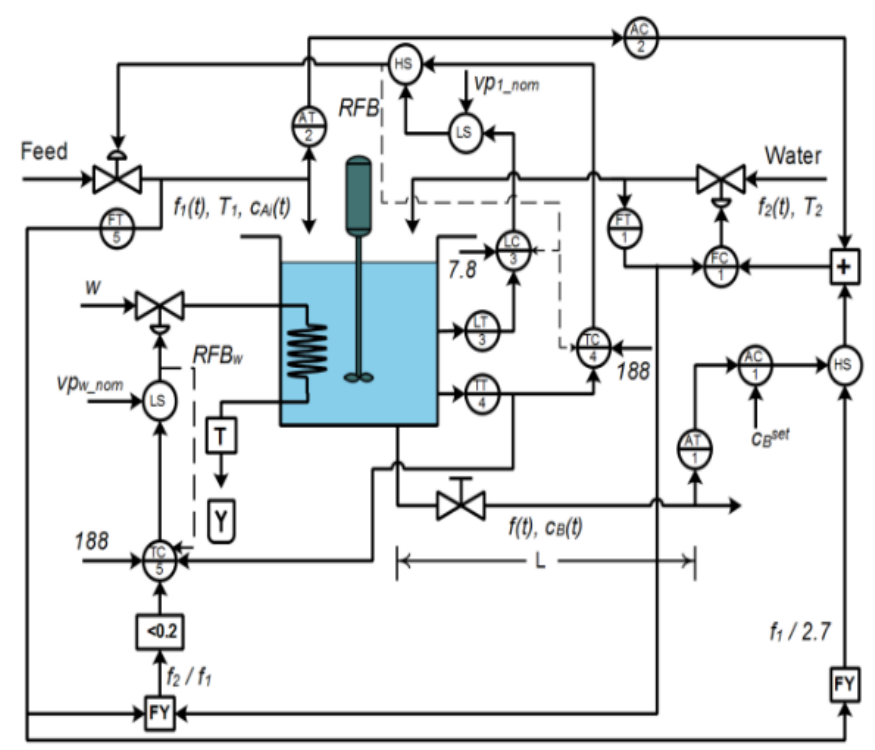

Figura 2. Estrategia de control para el reactor endotérmico. Fuente: Los autores. 
Tabla 2. Características de transmisores y elementos finales de control.

Fuente: Los autores.

\begin{tabular}{lll}
\hline Instrumento & Ganancia & $\begin{array}{l}\text { Constante de } \\
\text { tiempo (min) }\end{array}$ \\
\hline Transmisor de flujo corriente de & 2.0544 & $\approx 0$ \\
alimentación & 2.765 & $\approx 0$ \\
Transmisor de flujo corriente de agua & 50 & 0.8 \\
Transmisor de concentración & 4 & $\approx 0$ \\
Transmisor de nivel & 0.5 & 0.0017 \\
Transmisor de temperatura & 0.5893 & 0.1667 \\
Válvula vapor & 2.7056 & 0.1667 \\
Válvula corriente agua & 3.6414 & 0.1667 \\
Válvula corriente alimentación & & \\
\hline
\end{tabular}

Tabla 3. Modelos FOPDT.

\begin{tabular}{llll}
\hline Función de transferencia & $K$ & $\tau$ & $t_{0}$ \\
\hline$G_{1}(s)=C_{B}(s) / F_{2}(s)$ & -0.7148 & 5.6941 & 2.1478 \\
$G_{2}(s)=H(s) / M_{1}(s)$ & 0.8171 & 13.3877 & 0.0282 \\
$G_{3}(s)=T(s) / M_{1}(s)$ & -0.1291 & 7.4938 & 1.3762 \\
$G_{4}(s)=T(s) / W(s)$ & 0.1049 & 6.0868 & 0.3900 \\
$G_{5}(s)=C_{B}(s) / C_{A i}(s)$ & 21.4567 & 5.9390 & 2.5304 \\
\hline
\end{tabular}

Fuente: Los autores.

los parámetros con subíndice 5 corresponden a la función de trasferencia que representa el efecto de las perturbaciones $\mathrm{G}_{5}(\mathrm{~s})$ en la Tabla 3 , mientras que los parámetros con subíndice 5 corresponden a la función de trasferencia del proceso $\mathrm{G}_{1}(\mathrm{~s})$, de la misma Tabla 3.

\subsection{Control de nivel (Lazo 3)}

La función de transferencia empleada para la sintonía fue $G_{2}(s)$, en la que la variable manipulada es la posición de la válvula de la corriente de alimentación. Teniendo en cuenta la restricción que establece que, si el nivel del líquido aumenta por encima de 8 pies, el flujo másico total debe ser reducido, se estableció como set point de este controlador 7.5 pies.

\subsection{Control de temperatura (Lazo 4)}

La función de transferencia empleada para la sintonía fue $\mathrm{G}_{3}(\mathrm{~s})$, en la cual nuevamente la variable manipulada es la posición de la válvula de la corriente de alimentación (f1). Teniendo en cuenta la restricción que establece que, si la temperatura del reactor aumenta por encima de $190^{\circ} \mathrm{F}$, la relación agua/carga debe incrementarse, se estableció como set point de este controlador $188^{\circ} \mathrm{F}$.

En condiciones de operación normales (nivel y temperatura inferiores a 8 pies y $190^{\circ} \mathrm{F}$ respectivamente), la posición de la válvula de la corriente de alimentación es $\overline{v p}_{1}=37 \%$, no obstante cuando se presenta alguna de las condiciones de restricción, el controlador correspondiente debe tomar el mando con el fin de tratar de llevar el proceso a una zona de operación segura; es así como los controladores de los lazos 3 y 4 operan en sobre mando (Ribic et al., 2019; Smith \& Corripio, 2012), como se observa en la Figura 2.

\subsection{Control de temperatura (Lazo 5)}

En el lazo 4 se consideró el hecho que, si la temperatura del reactor aumenta por encima de $190^{\circ} \mathrm{F}$, la relación agua/carga debe incrementarse; no obstante, si dicha relación alcanza 5:1 y aun no se reduce la temperatura por debajo de $190^{\circ} \mathrm{F}$, debe reducirse el suministro de vapor. Es por esto por lo que se hizo necesario sintonizar un controlador de temperatura que manipule la posición de la válvula del flujo de vapor, para lo cual se emplearon los parámetros de la función de transferencia $\mathrm{G}_{4}(\mathrm{~s})$.

Este controlador solo toma el mando cuando se presenta la condición establecida $\left(\mathrm{T}>188^{\circ} \mathrm{F}\right.$ y f1/f 2 $<0.2$ ), en otro caso la posición de la válvula es $\overline{v p}_{w}=93 \%$.

\subsection{Control de relación}

La finalidad de este lazo de control es mantener la relación entre los flujos de alimentación y de agua por debajo de 3, (Javier Jiménez-Cabas et al., 2020; Mishra et al., 2020). Este sistema opera en 
sobremando con el control de concentración de B, entrando en operación una vez se haya detectado que la relación carga/agua es superior a 2.7, como se observa en la Figura 2.

La Tabla 4 muestra los parámetros de los distintos controladores sintonizados.

\section{Resultados obtenidos}

Con el objeto de verificar el desempeño de la estrategia de control diseñada, se realizaron varias simulaciones bajo condiciones de operación diferentes. La primera prueba realizada consistió en operar el sistema en condiciones nominales, haciendo $c_{B}^{\text {set }}=0.4$. La Figura 3 muestra el resultado obtenido. Se observa un sobrepico de $0.55 \%$ para la concentración de $\mathrm{B}$, que se puede considerar bastante bajo (rango de operación de la cromatografía de 0 a $2 \mathrm{lbmol} / \mathrm{ft}^{3}$, puede estar cercano a límites de detección de la técnica); en cuanto al tiempo de establecimiento se observó una reducción considerable, se pasó de 30 min en lazo abierto a 15 min en lazo cerrado; adicionalmente se logra un error en estado estacionario nulo con lo que se verifica la efectividad del lazo de control.

Tabla 4. Parámetros de los controladores empleados.

\begin{tabular}{cllll}
\hline Controlador & \multicolumn{1}{c}{$\boldsymbol{K}_{\boldsymbol{P}}$} & \multicolumn{1}{c}{$\boldsymbol{K}_{\boldsymbol{I}}$} & \multicolumn{1}{c}{$\boldsymbol{K}_{\boldsymbol{D}}$} & \multicolumn{1}{c}{$\boldsymbol{N}$} \\
\hline $\mathrm{FC} / 1$ & 1.4175 & 12.1251 & -0.0625 & 22.6864 \\
$\mathrm{AC} / 1$ & -2.6296 & -0.4342 & -1.1763 & 35.3144 \\
$\mathrm{LC} / 3$ & 4.5704 & 0.4189 & -7.3970 & 0.6179 \\
$\mathrm{TC} / 4$ & -28.2673 & -3.5556 & -7.0804 & 54.1931 \\
$\mathrm{TC} / 5$ & 26.3315 & 4.7671 & -16.5646 & 0.6944 \\
\hline
\end{tabular}

Fuente: Los autores.
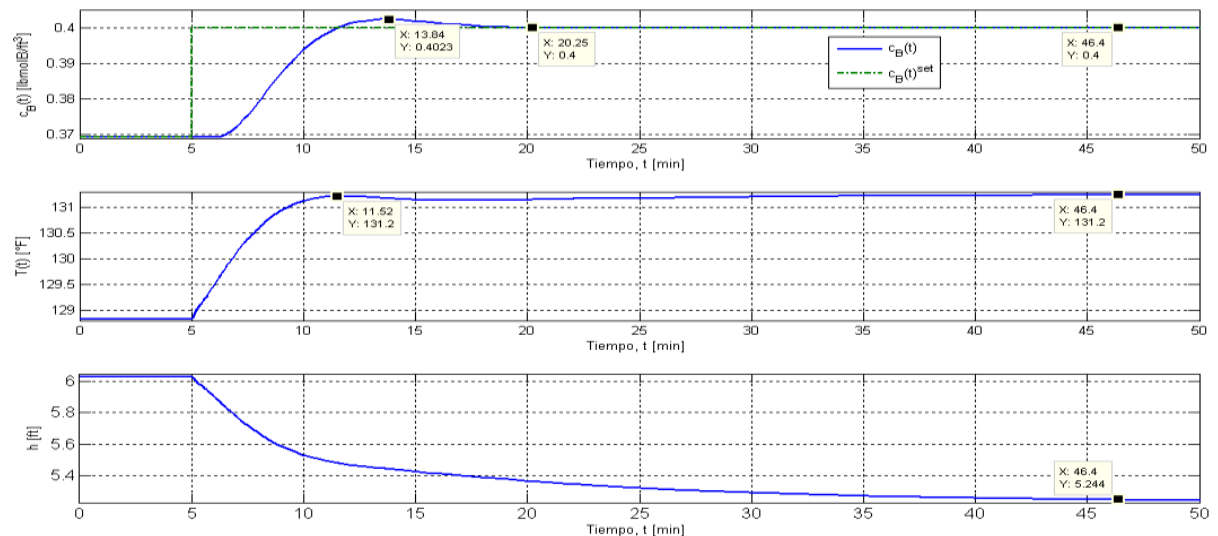

Figura 3. Respuesta al escalón en condiciones nominales.

Fuente: Los autores.
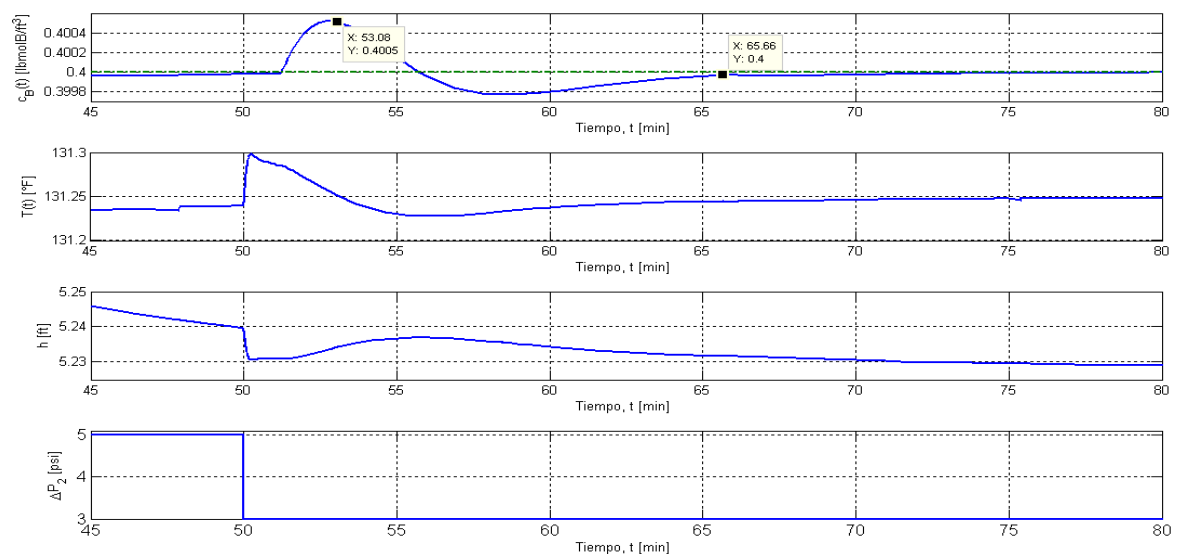

Figura 4. Respuesta ante perturbación en $\Delta \mathrm{P} 2$.

Fuente: Los autores. 
Por otra parte, se observa un incremento en la temperatura y una reducción en el nivel, debido a que para incrementar la concentración de $\mathrm{B}$ fue necesario reducir el flujo de agua entrante al reactor. La siguiente condición de operación considerada fue, una vez alcanzado el estado estacionario, considerar una variación de 2 psi en la caída de presión en la válvula de la corriente de agua.

Como se muestra en la Figura 4, se tiene una variación máxima del $0.125 \%$ en la concentración y el sistema retorna al valor del set point aproximadamente $17 \mathrm{~min}$ después de haberse presentado la perturbación, adicionalmente no se observan variaciones significativas para la temperatura y/o nivel del reactor.

Para el caso en que se presente una perturbación en la concentración de la corriente de alimentación, $c_{A i}$, como la mostrada en la Figura 5, se presenta una desviación máxima del $0.95 \%$ en la concentración de $\mathrm{B}$, pasados $20 \mathrm{~min}$ de haber aparecido la perturbación, el sistema muestra un error en estado estacionario del $0.025 \%$, el cual desaparece completamente $30 \mathrm{~min}$ después. Se observa una disminución en la temperatura y un incremento en el nivel, debidos al aumento en el flujo de agua entrante necesario para reducir la concentración de B.

Con el objeto de analizar el desempeño obtenido con el control de nivel en sobre mando, ante la situación extrema en que el nivel se acerque a 8 pies, se simuló el cierre de la válvula de salida del reactor (durante $130 \mathrm{~min}$ ), variando en un $30 \%$ el coeficiente de flujo de esta, como se muestra en la Figura 6. Se observa una desviación máxima del $2.925 \%$ en la concentración de B; a partir del momento en que se cierra la válvula le toma al sistema cerca de 103 min recuperarse, mientras que el tiempo de asentamiento a partir del momento en que desaparece la perturbación es de 23 min. Cabe mencionar que, sin el control de nivel, esta perturbación llevaría dicha variable a un valor cercano a los 12 pies.
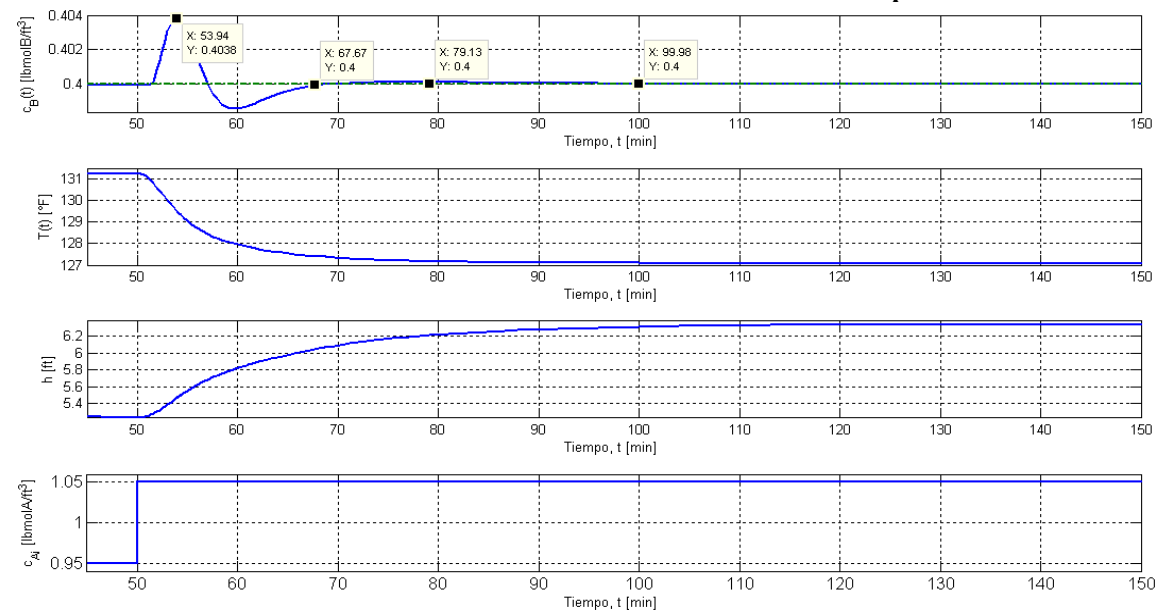

Figura 5. Respuesta ante perturbación en CAi.

Fuente: Los autores.
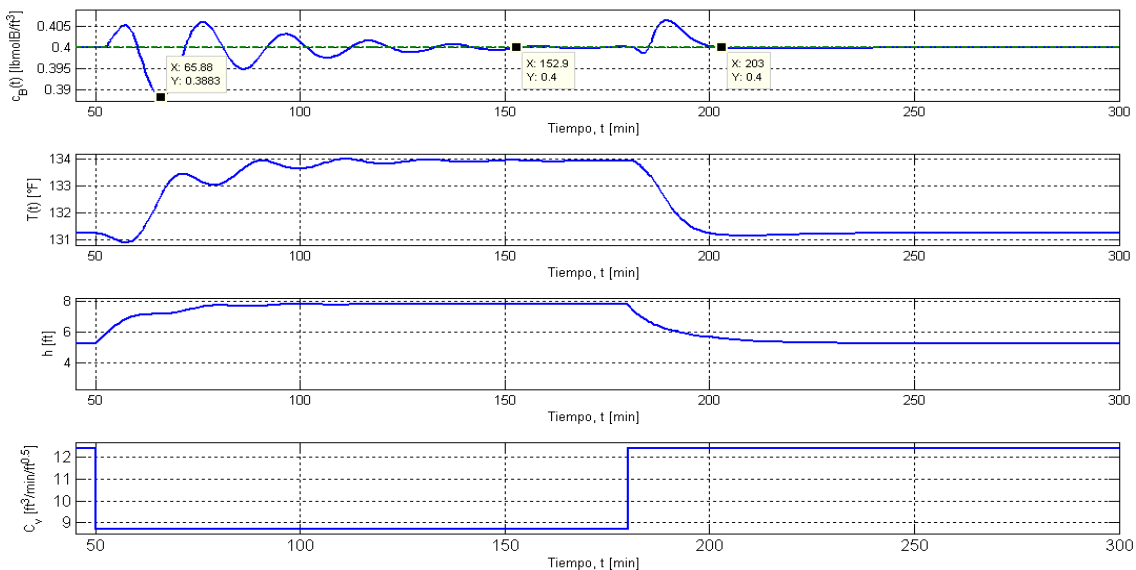

Figura 6. Respuesta ante perturbación en CV de la válvula de salida.

Fuente: Los autores. 

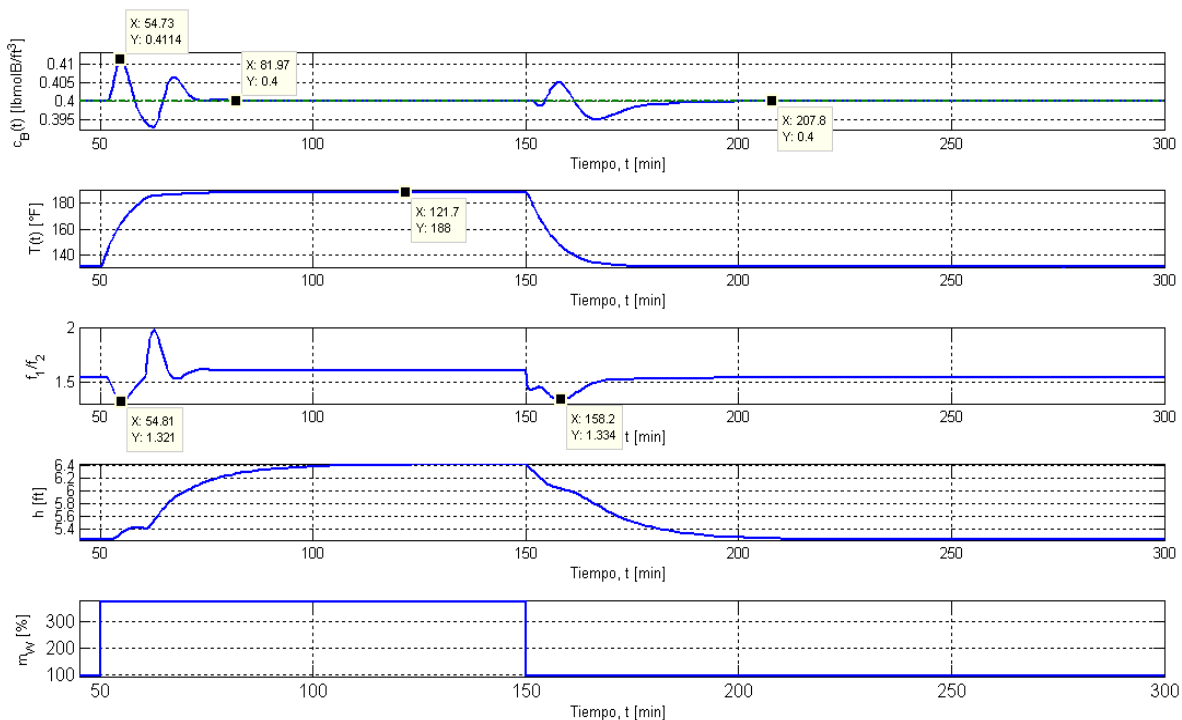

Figura 7. Respuesta ante perturbación en flujo de vapor.

Fuente: Los autores.
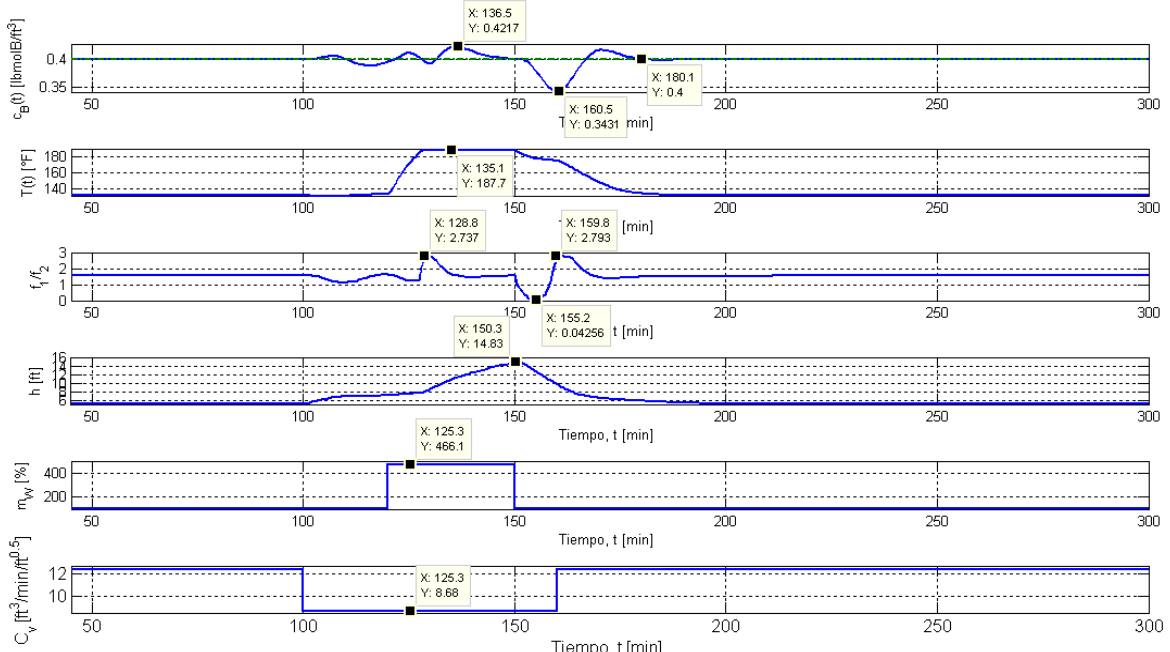

Figura 8. Respuesta ante perturbación en CV de la válvula de salida y flujo de vapor.

Fuente: Los autores.
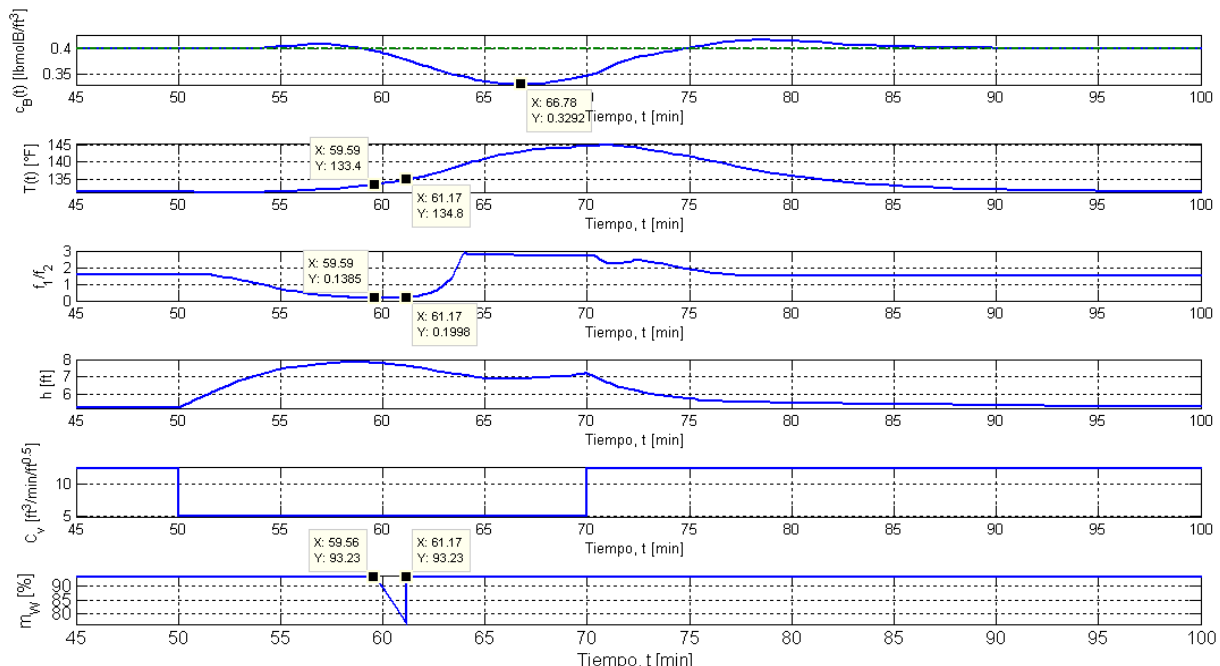

Figura 9. Respuesta ante perturbación en CV de la válvula de salida - Control temperatura.

Fuente: Los autores. 
Ahora, con el objeto de lograr la condición extrema para la temperatura se aplicó un incremento en el flujo de vapor tal como se muestra en la Figura 7. Cabe resaltar el desempeño del controlador de temperatura que no permite que dicha variable supere los $190^{\circ} \mathrm{F}$, sin este controlador la temperatura superaría los $200^{\circ} \mathrm{F}$. Adicionalmente se observa una desviación máxima del $2.85 \%$ en la concentración de B y un tiempo de establecimiento, a partir de la ocurrencia de la perturbación, de $32 \mathrm{~min}$. En los que a la relación carga/agua respecta, esta se mantiene dentro de los límites establecidos (0.2 - 3).

Por otra parte, para lograr una relación carga/agua superior a 3, y verificar el funcionamiento del control de relación, fue necesario aplicar simultáneamente dos de las perturbaciones ya analizadas, $\mathrm{CV}$ de la válvula de salida y flujo de vapor (ver Figura 8). Se observa que tanto el control de temperatura como el de relación se desempeñan correctamente al mantener sus variables controladas por debajo de los límites establecidos. Cabe mencionar que el control de relación hasta aquí propuesto solo garantiza que no se supere el límite superior de 3 más no el límite inferior de 0.2 .

En cuanto al nivel en el reactor, se observa que supera el límite de 8 pies, esto se debe a que el control de temperatura tiene una jerarquía mayor al de nivel en la estrategia propuesta y, en la condición de operación en estudio, dicho control toma el mando; ahora, dado que el nivel máximo del tanque reactor es de 25 pies (ver Tabla 1), no se presentan problemas de rebosamiento. Adicionalmente en la concentración de B se observa una desviación máxima del $-14.3 \%$ y un tiempo de asentamiento de $80 \mathrm{~min}$.

Finalmente, con el objeto de verificar el desempeño del sistema de control de temperatura que manipula el flujo del vapor, se simuló nuevamente una perturbación en el parámetro $\mathrm{CV}$ de la válvula de salida (ver Figura 9). El set point de este controlador debe ser $188^{\circ} \mathrm{F}$, como se mencionó antes, sin embargo, en vista de que la única forma encontrada para alcanzar la condición extrema de $\mathrm{T}$ $>190^{\circ} \mathrm{F}$ y f1/f2 < 0.2 fue incrementando el flujo de vapor (lo cual no podríamos hacer en este caso por ser la variable manipulada) y variando $\mathrm{C}_{\mathrm{V}}$, se optó por bajar dicho set point a $134^{\circ} \mathrm{F}$, solo para efectos de verificar el correcto funcionamiento del controlador en cuestión. Los resultados presentados en la Figura 9 muestran que durante el intervalo de tiempo en que la temperatura supera los $134^{\circ} \mathrm{F}$ y la relación carga/agua es inferior a 0.2 , el controlador de temperatura toma el mando y comienza a reducir el flujo de vapor como lo especifican las exigencias de diseño y una vez la relación carga/agua supera 0.2 , la posición de la válvula de vapor regresa a su valor nominal $\overline{v p}_{w}=93 \%$.

Por su parte la concentración de B presenta una desviación máxima del $-17.7 \%$ y un tiempo de recuperación cercano a los $100 \mathrm{~min}$. Las variables de nivel y temperatura se mantienen dentro de los rangos de operación permitidos.

\section{Conclusiones}

La estrategia de control propuesta cumple de manera adecuada las exigencias de desempeño.

El arreglo de ganancias relativas o RGA, se constituyó en una herramienta útil al momento de la selección de los pares de variables controlada/manipulada en el diseño de la estrategia de control del proceso estudiado.

La jerarquía seleccionada para los controladores que operan en sobre mando, permite mantener en valores adecuados las variables controladas.

Las técnicas avanzadas de control, como control en sobre mando, control por relación, cascada y feedforward, permitieron integrar controladores SISO para manejar este sistema multivariable.

Dado que las respuestas en las variables de salida del sistema, ante variaciones en las distintas entradas, presentaron comportamientos autorregulados y no oscilatorios, fue posible aproximar las dinámicas que las relacionan a modelos de primer orden más tiempo muerto, empleando la técnica de identificación Fit 3.

Las consideraciones en instrumentación arrojan valores acordes al funcionamiento del proceso, por lo tanto, concluimos que son adecuadas.

La respuesta a las diferentes perturbaciones consideradas es adecuada, las desviaciones con respecto al set point establecido son bastante bajas para casi todos los casos. Para las condiciones 
especiales donde se aumentó la temperatura a través del flujo de vapor se obtuvieron desviaciones en la concentración de B mayores al 10\%, no obstante, cabe resaltar que estas condiciones de operación no son alcanzables en condiciones reales de operación.

\section{Referencias}

Druzgalski, C. L., Ashby, A., Guss, G., King, W. E., Roehling, T. T., \& Matthews, M. J. (2020). Process optimization of complex geometries using feed forward control for laser powder bed fusion additive manufacturing. Additive Manufacturing, 101169.

Jiménez-Cabas, J., Meléndez-Pertuz, F., OvallosGazabon, D., Vélez-Zapata, J., Castellanos, $H$. E., Cárdenas, C. A., Sánchez, J. F., Jimenez, G., Mora, C., Sanz, F. A., \& Collazos, C. A. (2019). Robust Control of an Evaporator Through Algebraic Riccati Equations and D-K Iteration. In Lecture Notes in Computer Science (including subseries Lecture Notes in Artificial Intelligence and Lecture Notes in Bioinformatics): Vol. 11620 LNCS. https://doi.org/10.1007/978-3030-24296-1_58

Jiménez-Cabas, Javier, Meléndez-Pertuz, F., DíazCharris, L. D., Collazos-Morales, C., \& González, R. E. R. (2020). Robust Control of the Classic Dynamic Ball and Beam System. International Conference on Computational Science and Its Applications, 134-144.

Mishra, P., Kumar, V., \& Rana, K. P. S. (2020). A nonlinear framework for stiction compensation in ratio control loop. ISA Transactions.

Ribic, A., Crair, M. C., \& Biederer, T. (2019). Synapse-selective control of cortical maturation and plasticity by parvalbumin-autonomous action of SynCAM 1. Cell Reports, 26(2), 381393.

Smith, C. A., \& Corripio, A. B. (2012). Principles and Practice of Automatic Process Control. Editorial Félix Varela.

Vijayan, A. T., \& Ashok, S. (2020). Relative Array Analysis of Visual Servo System. 2020 7th International Conference on Signal Processing and Integrated Networks (SPIN), 117-121. 